International Journal of Pure and Applied Mathematics

Volume 98 No. 1 2015, 157-168

ISSN: 1311-8080 (printed version); ISSN: 1314-3395 (on-line version)

url: http://www.ijpam.eu

doi: http://dx.doi.org/10.12732/ijpam.v98i1.13

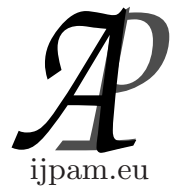

\title{
SIGNED CLIQUE EDGE-DOMATIC NUMBER OF A GRAPH
}

\author{
Zhang Li-Xian ${ }^{1}$, Lu Xin-Zhong ${ }^{2}$ \\ ${ }^{1,2}$ Department of Mathematics \\ Zhejiang Normal University \\ Zhejiang, P.R. CHINA
}

\begin{abstract}
In this paper, the problem of signed clique edge-domatic number is considered for any finite,nonempty and simple graph $G$ with the maximal clique $K_{\omega}$. At first,using induction method, we study the signed clique edge-domatic number for a class of graphs with maximal clique $K_{\omega}(\omega=2,3)$. By inducing and summarizing, we obtain that the signed clique edge-domatic number on this class of graphs and the signed clique edge-domatic number of their maximal clique $K_{\omega}$ have some relations. Inspired by ways and regulars of the proof of graphs with $\omega(G)=2,3$, we take seriously to study the signed clique edge-domatic number for any graph $G$ with $K_{\omega}(\omega \geq 4)$ and have proved that the signed clique edge-domatic numbers on $G$ and on $K_{\omega}(\omega \geq 4)$ are equal, so we achieve the signed clique edge-domatic number for any graph $G$. This conclusion is a practical significance for the promotion of signed edge-domatic number.
\end{abstract}

AMS Subject Classification: 05C69

Key Words: clique, signed clique edge domination, signed clique edgedomatic number, signed clique edge dominating family

\section{Introduction}

In this paper, all of the graphs that we consider are finite,nonempty, simple and undirected. Let $G=(V(G), E(G))$ be a graph with vertex set $V(G)$ and edge set $E(G)$. The order $n$ of $G$ denotes the number of vertices of $G$ and the size $m$ of $G$ denotes the number of edges of $G$. For any edge $e \in E(G), N_{G}(e)$ will

Received: September 21, 2014

(C) 2015 Academic Publications, Ltd. url: www.acadpubl.eu

${ }^{\S}$ Correspondence author 
denote the open neighborhood of $e$ in $G$ and $N_{G}[e]=N_{G}(e) \bigcup\{e\}$ will denote the closed neighborhood.

Definition 1. (see [1]) A function $f^{\prime}: E(G) \rightarrow\{-1,1\}$ is called the signed edge dominating function (SEDF) of $\mathrm{G}$ if $\sum_{e^{\prime} \in N[e]} f^{\prime}\left(e^{\prime}\right) \geq 1$, for every $e \in E(G)$. The signed edge domination number of $G$ is defined as: $\gamma_{s}^{\prime}(G)=$ $\min \left\{\sum_{e \in E(G)} f^{\prime}(e) \mid f\right.$ is an SEDF of $\left.\mathrm{G}\right\}$.

Every complete subgraph $K_{\omega}$ of $G$ that is not included by other any complete subgraph of $G$ is called a maximal clique of $G$. The order of maximal complete subgraph is called clique number of $G$, which is denoted by $\omega(G)$.

Definition 2. (see [2]) A function $f^{\prime}: E(G) \rightarrow\{-1,1\}$ is called a signed clique edge dominating function(SCEDF) of $G$ if $\sum_{e \in E\left(K_{\omega}\right)} f^{\prime}(e) \geq 1$,for every maximal clique $K_{\omega}$ of $G$. Let $F_{\text {sced }}(G)$ is a set of all signed clique edge dominating functions on $G$. The signed clique edge domination number of $G$ is defined as: $\gamma_{s c l}^{\prime}(G)=\min \left\{\sum_{e \in E(G)} f^{\prime}(e) \mid f^{\prime} \in F_{\text {sced }}(G)\right\}$.

If $S \subseteq E=E(G)$, we denote

$$
f^{\prime}(S)=\sum_{e \in S} f^{\prime}(e)
$$

then $\gamma_{s c l}^{\prime}(G)=\min \left\{f^{\prime}(E(G)) \mid f^{\prime} \in F_{\text {sced }}(G)\right\}$. We have concluded some properties of $\gamma_{s c l}^{\prime}(G)$ based on its definition:

(a) $\gamma_{s c l}^{\prime}(G)=|E(G)|$ if and only if $G$ has no triangles.

(b) For arbitrary two disjoint graphs $G_{1}, G_{2}$, there is

$$
\gamma_{s c l}^{\prime}\left(G_{1} \bigcup G_{2}\right)=\gamma_{s c l}^{\prime}\left(G_{1}\right)+\gamma_{s c l}^{\prime}\left(G_{2}\right)
$$

Definition 3. (see [3]) A set $\left\{f_{1}^{\prime}, f_{2}^{\prime}, \cdots, f_{d}^{\prime}\right\}$ of signed edge dominating functions on $\mathrm{G}$ with the property that $\sum_{i=1}^{d} f_{i}^{\prime}(e) \leq 1$ for each edge $e \in E(G)$ is called a signed edge dominating family on $\mathrm{G}$. The maximum number of functions in a signed edge dominating family on $\mathrm{G}$ is the signed edge-domatic number of $\mathrm{G}$, denoted by $d_{s}^{\prime}(G)$. 
Now we similarly define a signed clique edge-domatic number of $G$.

Definition 4. Let a $\operatorname{set}\left\{f_{1}^{\prime}, f_{2}^{\prime}, \cdots, f_{d}^{\prime}\right\} \subseteq F_{\text {sced }}(G)$ on $\mathrm{G}$ with the property that $\sum_{i=1}^{d} f_{i}^{\prime}(e) \leq 1$ for each edge $e \in E(G)$ be called a signed clique edge dominating family on G. Use $\Psi(G)$ to denote the collection of families of signed clique edge-dominations on $G$. The signed clique edge-domatic number of $\mathrm{G}$ is denoted by $d_{s c l}^{\prime}(G)=\max \{|F|: F \in \Psi(G)\}$. Obviously, $d_{s c c l}^{\prime}(G) \geq 1$.

V. Lutz, B. Zelinka study the signed domatic number and they give some new ways to solve those problems in [6] and [7], then X.J.Li and J.M.Xu research the signed edge domatic number in [3] and B.Xu proves the signed cycle domination number on graphs in [8]. So this article mainly studys the signed clique edge-domatic number.

\section{Basic Properties of the Signed Clique Edge-domatic Number of a Graph}

Theorem 1. Let $G$ be a graph of size $|E(G)|=m$ with the signed clique edge domination number $\gamma_{s c l}^{\prime}(G)$ and the signed clique edge-domatic number $d_{s c l}^{\prime}(G)$, then $\gamma_{s c l}^{\prime}(G) d_{s c l}^{\prime}(G) \leq m$.

Proof. If $\left\{f_{1}^{\prime}, f_{2}^{\prime}, \cdots, f_{d}^{\prime}\right\} \in \Psi(G)$ such that $d=d_{s c l}^{\prime}(G)$, then the definitions imply:

$$
\begin{aligned}
\gamma_{s c l}^{\prime}(G) d_{s c l}^{\prime}(G)=\gamma_{s c l}^{\prime}(G) d=\gamma_{s c l}^{\prime}(G) \sum_{i=1}^{d} 1 & \leq \sum_{i=1}^{d} \sum_{e \in E(G)} f_{i}^{\prime}(e) \\
& =\sum_{e \in E(G)} \sum_{i=1}^{d} f_{i}^{\prime}(e) \leq \sum_{e \in E(G)} 1=m .
\end{aligned}
$$

Theorem 2. For an arbitrary graph $G, d_{s c l}^{\prime}(G)$ is an odd integer.

Proof. For an arbitrary graph $G$, suppose that $d=d_{s c l}^{\prime}(G)$ is an even number. Let $\left\{f_{1}^{\prime}, f_{2}^{\prime}, \cdots, f_{d}^{\prime}\right\} \in \Psi(G)$ be the corresponding signed clique edge dominating family on $G$. We have $\sum_{i=1}^{d} f_{i}^{\prime}(e) \leq 1$ for each $e \in E(G)$ based on definition. But on the left-hand side of this inequality a sum of an even 
number of odd summands occurs. Therefore,it is an even number and we obtain $\sum_{i=1}^{d} f_{i}^{\prime}(e) \leq 0$ for each $e \in E(G)$.This forces:

$$
d=\sum_{i=1}^{d} 1 \leq \sum_{i=1}^{d} \sum_{e \in E(K \omega)} f_{i}^{\prime}(e)=\sum_{e \in E(K \omega)} \sum_{i=1}^{d} f_{i}^{\prime}(e) \leq 0
$$

which contradicts $d_{s c c l}^{\prime}(G) \geq 1$.

Theorem 3. If $G$ is a graph with maximal clique $K_{\omega}, \omega \geq 2$, then

$$
d_{s c l}^{\prime}(G) \leq\left\{\begin{array}{lllll}
\frac{\omega(\omega-1)}{4} & \text { if } & \omega \equiv 0 & \text { or } & 1(\bmod 4) \\
\frac{\omega(\omega-1)}{2} & \text { if } & \omega \equiv 2 & \text { or } & 3(\bmod 4)
\end{array}\right.
$$

Proof. Let the size of a maximal clique $K_{\omega}$ on $G$ be $\left|E\left(K_{\omega}\right)\right|=\frac{\omega(\omega-1)}{2}$. Let $d=d_{s c l}^{\prime}(G),\left\{f_{1}^{\prime}, f_{2}^{\prime}, \cdots, f_{d}^{\prime}\right\} \in \Psi(G)$ be the corresponding signed clique edge dominating family on $G$. Now we have two cases for the size of a maximal clique on $G$.

Case $1 . \omega \equiv 0$ or $1(\bmod 4)$.

If $\omega \equiv 0$ or $1(\bmod 4),\left|E\left(K_{\omega}\right)\right|=\frac{\omega(\omega-1)}{2}$ is an even number. Let $f_{i}^{\prime} \in$ $\left\{f_{1}^{\prime}, f_{2}^{\prime}, \cdots, f_{d}^{\prime}\right\}$, then $f_{i}^{\prime}\left(E\left(K_{\omega}\right)\right) \geq 1$. Therefore, $f_{i}^{\prime}\left(E\left(K_{\omega}\right)\right) \geq 2$. The imply:

$$
2 d=\sum_{i=1}^{d} 2 \leq \sum_{i=1}^{d} \sum_{e \in E\left(K_{\omega}\right)} f_{i}^{\prime}(e)=\sum_{e \in E\left(K_{\omega}\right)} \sum_{i=1}^{d} f_{i}^{\prime}(e) \leq \sum_{e \in E\left(K_{\omega}\right)} 1=\frac{\omega(\omega-1)}{2}
$$

so $d=d_{s c l}^{\prime}(G) \leq \frac{\omega(\omega-1)}{4}$.

Case $2 . \omega \equiv 2$ or $3(\bmod 4)$.

If $\omega \equiv 2$ or $3(\bmod 4)$, then $\left|E\left(K_{\omega}\right)\right|=\frac{\omega(\omega-1)}{2}$ is an odd number. Let $f_{i}^{\prime} \in\left\{f_{1}^{\prime}, f_{2}^{\prime}, \cdots, f_{d}^{\prime}\right\}$,then $f_{i}^{\prime}\left(E\left(K_{\omega}\right)\right) \geq 1$. This imply:

$$
d=\sum_{i=1}^{d} 1 \leq \sum_{i=1}^{d} \sum_{e \in E\left(K_{\omega}\right)} f_{i}^{\prime}(e)=\sum_{e \in E\left(K_{\omega}\right)} \sum_{i=1}^{d} f_{i}^{\prime}(e) \leq \sum_{e \in E\left(K_{\omega}\right)} 1=\frac{\omega(\omega-1)}{2},
$$

so $d=d_{s c l}^{\prime}(G) \leq \frac{\omega(\omega-1)}{2}$.

Theorem 4. Let $G$ be a graph of size $m$ with no triangles. Then $d_{s c l}^{\prime}(G)=$ 1. 
Proof. We can know the value of the signed clique edge domination number on $G$ from its properties(a). We have $\gamma_{s c l}^{\prime}(G)=|E(G)|=m$.

Following from theorem $1, \gamma_{s c l}^{\prime}(G) d_{s c l}^{\prime}(G) \leq m$, we can obtain $d_{s c l}^{\prime}(G) \leq$ $\frac{m}{|E(G)|}=1$. Since $d_{s c l}^{\prime}(G) \geq 1$, the equality can be achieved: $d_{s c l}^{\prime}(G)=1$.

Corollary 1. For any tree $T$ of order $n \geq 3$, then $d_{s c l}^{\prime}(T)=1$.

A plane graph $G$ is called a maximal plane graph if its every face is a triangle.

Lemma 1. For any maximal plane graph $G$ of order $n(n \geq 3)$, then it has $2 n-4$ faces (including its outside boundary), see [2].

Lemma 2. For any maximal planar graph $G$ of order $n(n \geq 3)$, then $\gamma_{s c l}^{\prime}(G) \geq n-2$.

Proof. For any maximal planar graph $G$ of order $n(n \geq 3)$ has $2 n-4$ faces(including its outside boundary) from Lemma 1.

Let $F_{1}, F_{2}, \cdots, F_{2 n-4}$ be all faces of $G$ and $E\left(F_{i}\right)$ be a set of all edges of the face $F_{i}$.It is not hard to know that every face $F_{i}$ is a maximal clique of $G$. Let $f^{\prime} \in F_{\text {sced }}(G)$ satisfy $\gamma_{s c l}^{\prime}(G)=f^{\prime}(E(G))$, then $f^{\prime}\left(E\left(F_{i}\right)\right) \geq 1$ for any face $F_{i}$, based on definition of the function $f^{\prime}$,so

$$
2 \gamma_{s c l}^{\prime}(G)=\sum_{i=1}^{2 n-4} \sum_{e \in E\left(F_{i}\right)} f^{\prime}(e) \geq 2 n-4, \text { so } \gamma_{s c l}^{\prime}(G) \geq n-2 .
$$

Next we will introduce some class of graphs with some properties such that equality can be achieved at above.

Let $G_{1}=K_{3}, G_{m}$ is the graph obtained from $G_{m-1}$ by adding a vertex $u$ and three edges $u v_{1}, u v_{2}, u v_{3}$, where $v_{1}, v_{2}, v_{3}$ are three vertices on the same inner face(triangle) of $G_{m-1}(m=2,3, \cdots)$. Obviously, $G_{m}$ is a maximal planar graph,and $\left|V\left(G_{m}\right)\right|=m+2$.

Lemma 3. Let $M=\left\{G_{1}, G_{2}, \cdots, G_{m}, \cdots\right\}$, then $\gamma_{s c l}^{\prime}(G)=n-2$ for any graph $G \in M$ with order $n$.

Proof. Obviously, for any graph $G \in M$ with order $n(n \geq 3)$,by Lemma $2, \gamma_{s c l}^{\prime}(G) \geq n-2$. Next we can obtain $\gamma_{s c l}^{\prime}(G) \leq n-2$.

Using induction on order $n$ of $G$, we prove that $f^{\prime}(E(G)) \leq n-2$ holds for all $n(n=3,4,5, \cdots)$. 
Clearly,when $n=3$, we let $G=K_{3}$, then $G$ is the maximal clique for itself.Let $E(G)=\left\{e_{1}, e_{2}, e_{3}\right\}$ and $f^{\prime}(E(G))=\gamma_{s c l}^{\prime}(G)$, there exist exactly values of two edges 1 , so we can suppose that $f^{\prime}\left(e_{1}\right)=1, f^{\prime}\left(e_{2}\right)=1, f^{\prime}\left(e_{3}\right)=-1$. Obviously, $f^{\prime}(E(G)) \leq n-2$ is true.

Suppose that there exists an SCEDF, say $g^{\prime}$ on $G=G_{n-3} \in M$ with order $n-1$ such that $g^{\prime}(E(G)) \leq(n-1)-2$ is true. Then we consider $G=G_{n-2} \in M$. Based on the definition of graph $G$, we can know that there exists 3-degree vertex $v$ in some inner face of $G=G_{n-2}$. Let $H=G-\{v\} \in M$, then by the induction hypothesis, there is $g^{\prime}(E(H)) \leq(n-1)-2$. Let $S=$ $N_{G}(v)=\left\{u_{1}, u_{2}, u_{3}\right\}$, then $H[S]=K_{3}$ and the values of three edges of $H[S]$ exactly two are 1 . Without loss of generality, let $g^{\prime}\left(u_{1} u_{2}\right)=-1, g^{\prime}\left(u_{2} u_{3}\right)=1$ and $g^{\prime}\left(u_{1} u_{3}\right)=1$. When the vertex $v$ adds to $H[S]=K_{3}$, it forms three sets $S_{1}=\left\{u_{1}, u_{2}, v\right\}, S_{1}=\left\{u_{1}, u_{3}, v\right\}, S_{1}=\left\{u_{3}, u_{2}, v\right\}$, which constitute three maximal inner faces of $G=G_{n-2}$. Define a function $f^{\prime}$ on $G=G_{n-2}$ as follows:

$$
f^{\prime}(e)=\left\{\begin{array}{cll}
g^{\prime}(e) & \text { if } & e \in E(G-\{v\}) \\
1 & \text { if } & e \in\left\{u_{1} v, u_{2} v\right\} \\
-1 & \text { if } & e=u_{3} v
\end{array}\right.
$$

It is not hard to verify that $f^{\prime}$ is an SCEDF of $G=G_{n-2}$ such that $f^{\prime}(E(G))=2-1+f^{\prime}(E(H)) \leq n-2$ is true. So $f^{\prime}(E(G))=n-2$.

Theorem 5. Let $M=\left\{G_{1}, G_{2}, \cdots, G_{m}, \cdots\right\}$, for any graph $G$ in $M$, then $d_{s c l}^{\prime}(G)=3$.

Proof. For any graph $G$ with the order $n$ in $M$, the number of the maximal cliques on $G$ is $2 n-4$ by Lemma 1 . Let $F_{1}, F_{2}, \cdots, F_{2 n-4}$ denotes all maximal cliques of $G$. By theorem1, we determine the signed clique edge-domatic number $d_{s c l}^{\prime}(G) \leq 3$, since the size of $G$ is $|E(G)|=3 n-6$. Therefore, we can get $d_{s c l}^{\prime}(G)=1$ or 3 by theorem 1 and theorem 2 . Now we prove that $d_{s c l}^{\prime}(G)=3$ is right.

Using induction on order $n$ of $G$, we prove that $d_{s c l}^{\prime}(G)=3$ holds for all $n(n=3,4,5, \cdots)$.

When $n=3$, then $G=K_{3}=G_{1}$, so it is easy to verify that $d_{s c l}^{\prime}(G)=3$ is true.

When $|V(G)|=n-1$, then $G=G_{n-3}$, so we suppose that $d_{s c l}^{\prime}(G)=3$ is also true. Let $F_{1}, F_{2}, \cdots, F_{2(n-1)-4}$ denote all the faces of $G_{n-3}$ and $\left\{g_{1}^{\prime}, g_{2}^{\prime}, g_{3}^{\prime}\right\} \in$ $\Psi\left(G_{n-3}\right)$. Based on the definition of graph $G=G_{n-2}$, we can add the vertex $u$ to any inner face $F_{i}$ of $G_{n-3}$ such that $u$ is adjacent to three vertices of $F_{i}$. Let $V\left(F_{i}\right)=\left\{v_{1 i}, v_{2 i}, v_{3 i}\right\}$, so we can get additional three inner faces $F_{1 i}, F_{2 i}, F_{3 i}$ 
which are formed by $\left\{v_{1 i}, v_{2 i}, u\right\},\left\{v_{2 i}, v_{3 i}, u\right\},\left\{v_{3 i}, v_{1 i}, u\right\}$.

Therefore, $F_{1 i}, F_{2 i}, F_{3 i}$ are inner faces of $G_{n-2}$. Define $f_{1}^{\prime}, f_{2}^{\prime}, f_{3}^{\prime}$ on $G_{n-2}$ as following:

When $g_{1}^{\prime}\left(v_{1 i} v_{2 i}\right)=-1$, there must be $g_{1}^{\prime}\left(v_{1 i} v_{3 i}\right)=g_{1}^{\prime}\left(v_{2 i} v_{3 i}\right)=1$. Let

$$
f_{1}^{\prime}(e)=\left\{\begin{array}{cll}
g_{1}^{\prime}(e) & \text { if } & e \in E\left(G_{n-2}-\{u\}\right) \\
1 & \text { if } & e \in\left\{u v_{1 i}, u v_{2 i}\right\} \\
-1 & \text { if } & e=u v_{3 i}
\end{array}\right.
$$

When $g_{2}^{\prime}\left(v_{2 i} v_{3 i}\right)=-1$, there must be $g_{2}^{\prime}\left(v_{1 i} v_{3 i}\right)=g_{2}^{\prime}\left(v_{1 i} v_{2 i}\right)=1$. Let

$$
f_{2}^{\prime}(e)=\left\{\begin{array}{cll}
g_{2}^{\prime}(e) & \text { if } & e \in E\left(G_{n-2}-\{u\}\right) \\
1 & \text { if } & e \in\left\{u v_{2 i}, u v_{3 i}\right\} \\
-1 & \text { if } & e=u v_{1 i}
\end{array}\right.
$$

When $g_{3}^{\prime}\left(v_{1 i} v_{3 i}\right)=-1$, there must be $g_{3}^{\prime}\left(v_{1 i} v_{2 i}\right)=g_{3}^{\prime}\left(v_{2 i} v_{3 i}\right)=1$. Let

$$
f_{3}^{\prime}(e)=\left\{\begin{array}{cll}
g_{3}^{\prime}(e) & \text { if } & e \in E\left(G_{n-2}-\{u\}\right) \\
1 & \text { if } & e \in\left\{u v_{1 i}, u v_{3 i}\right\} \\
-1 & \text { if } & e=u v_{2 i}
\end{array}\right.
$$

It is not hard to verify that $\left\{f_{1}^{\prime}, f_{2}^{\prime}, f_{3}^{\prime}\right\} \in \Psi\left(G_{n-2}\right)$, so $d_{s c l}^{\prime}(G)=d_{s c l}^{\prime}\left(G_{n-2}\right) \geq$ 3.Therefore, $d_{s c l}^{\prime}(G)=3$.

Lemma 4. For any graph $G$ with size $m$, let $\omega(G)=\omega(\omega \geq 4)$ and the number of maximal cliques of $G$ be $\alpha(\alpha \geq 1)$, then $d_{s c l}^{\prime}(G)=d_{s c l}^{\prime}\left(K_{\omega}\right)$.

Proof. Let $K_{\omega} \in\left\{K_{\omega_{1}}, K_{\omega_{2}}, \cdots, K_{\omega_{\alpha}}\right\}$ be any maximal clique of $G$, then we can know $\left|V\left(K_{\omega_{i}}\right)\right|=\omega, i=1,2, \cdots, \alpha$.

Let $E_{1}=E\left(K_{\omega_{1}}\right) \bigcup E\left(K_{\omega_{2}}\right) \bigcup \cdots \bigcup E\left(K_{\omega_{\alpha}}\right), E_{2}=E(G) \backslash E_{1}$. Let $G_{1}=$ $G\left[E_{1}\right]$ be an induced subgraph of edges $E_{1}$ in $G$.

We can prove this lemma from two aspects next.

Let $\left\{g_{1 j}^{\prime}, g_{2 j}^{\prime}, \cdots, g_{d_{j} j}^{\prime}\right\} \in \Psi\left(K_{\omega_{j}}\right), j=1,2,3, \cdots, \alpha$, then we can get $d_{1}=$ $d_{2}=\cdots=d_{j}=d=d_{s c l}^{\prime}\left(K_{\omega}\right)$, since $K_{\omega_{1}}, K_{\omega_{2}}, \cdots, K_{\omega_{\alpha}}$ are maximal cliques of $G$. Now we consider two cases as following.

Case 1. Let $E\left(K_{\omega_{i}}\right) \cap E\left(K_{\omega_{j}}\right)=\emptyset$, if $i \neq j$ for any $i, j=1,2, \cdots, \alpha$.

Let $f_{1 j}^{\prime}, f_{2 j}^{\prime}, \cdots, f_{d j}^{\prime}, j=1,2, \cdots, \alpha$ be functions on $G$ such that satisfying :

$$
f_{i j}^{\prime}(e)=\left\{\begin{array}{cll}
g_{i j}^{\prime}(e) & \text { if } & e \in E\left(K_{\omega_{j}}\right), i=1,2, \cdots, d \\
0 & \text { if } & e \in E(G) \backslash E\left(K \omega_{j}\right)
\end{array}\right.
$$


Let $F_{1}^{\prime}, F_{2}^{\prime}, \cdots, F_{d}^{\prime}$ be functions on $G$ such that

$$
F_{i}^{\prime}(e)=\left\{\begin{array}{cll}
\sum_{j=1}^{\alpha} f_{i j}^{\prime}(e) & \text { if } & e \in E_{1}, i=1,2, \cdots, d \\
-1 & \text { if } & e \in E(G) \backslash E_{1}
\end{array}\right.
$$

It is not hard to verify that $\left\{F_{1}^{\prime}, F_{2}^{\prime}, \cdots, F_{d}^{\prime}\right\} \in \Psi(G)$, then $d_{s c l}^{\prime}(G) \geq d=$ $d_{s c l}^{\prime}\left(K_{\omega}\right)$.

Case 2. Let $E\left(K_{\omega_{i}}\right) \bigcap E\left(K_{\omega_{j}}\right) \neq \emptyset$, if $i \neq j$ for some $i, j=1,2, \cdots, \alpha$.

Let $E_{1}=\left\{e_{1 \beta_{1}}, e_{2 \beta_{2}}, \cdots, e_{\left|E_{1}\right| \beta_{\left|E_{1}\right|}}\right\}$, where $e_{h \beta_{h}} \in E_{1}(h=1,2, \cdots$, $\left.\left|E_{1}\right|, \beta_{h}=1,2, \cdots, \alpha\right)$ is an edge that appears in $\beta_{h}$ cliques of $G$. For some $k, j, k \neq j$, let $E\left(K_{\omega_{j}}\right) \bigcap E\left(K_{\omega_{k}}\right)=\left\{e_{1}, e_{2}, \cdots, e_{s}\right\}, s \geq 1$. Since $K_{\omega_{1}}, K_{\omega_{2}}, \cdots$, $K_{\omega_{\alpha}}$ are maximal cliques, there must exist functions $g_{i j}^{\prime}, g_{i k}^{\prime}$ such that $g_{i j}^{\prime}(e)=$ $g_{i k}^{\prime}(e)$ for every $e \in E\left(K_{\omega_{j}}\right) \bigcap E\left(K_{\omega_{k}}\right)$.

Let $f_{1 j}^{\prime}, f_{2 j}^{\prime}, \cdots, f_{d j}^{\prime}, j=1,2, \cdots, \alpha$ be functions on $G$ such that satisfying :

$$
f_{i j}^{\prime}(e)=\left\{\begin{array}{cll}
g_{i j}^{\prime}\left(e_{h \beta_{h}}\right) / \beta_{h} & \text { if } & e=e_{h \beta_{h}} \in E\left(K_{\omega_{j}}\right), i=1,2, \cdots, d \\
0 & \text { if } & e \in E(G) \backslash E\left(K_{\omega_{j}}\right)
\end{array}\right.
$$

Let $F_{1}^{\prime}, F_{2}^{\prime}, \cdots, F_{d}^{\prime}$ be functions on $G$ such that

$$
F_{i}^{\prime}(e)=\left\{\begin{array}{cll}
\sum_{j=1}^{\alpha} f_{i j}^{\prime}(e) & \text { if } & e \in E_{1}, i=1,2, \cdots, d \\
-1 & \text { if } & e \in E(G) \backslash E_{1}
\end{array}\right.
$$

It is not hard to verify that $\left\{F_{1}^{\prime}, F_{2}^{\prime}, \cdots, F_{d}^{\prime}\right\} \in \Psi(G)$, then $d_{s c l}^{\prime}(G) \geq d=$ $d_{s c l}^{\prime}\left(K_{\omega}\right)$.

On the other hand, let $\left\{f_{1}^{\prime}, f_{2}^{\prime}, \cdots, f_{d}^{\prime}\right\} \in \Psi(G)$ and $d=d_{s c l}^{\prime}(G)$. Therefore, there exist $f_{i}^{\prime}\left(E\left(K_{\omega_{j}}\right)\right) \geq 1$ for every $K_{\omega_{j}}, j=1,2, \cdots, \alpha$ and $\sum_{i=1}^{d} f_{i}^{\prime}(e) \leq 1$ for each $e \in E(G)$. Let $g_{k}^{\prime}: E_{1} \rightarrow\{-1,1\}, k=1,2, \cdots, d$ be a function of $G_{1}$ and satisfy: $g_{i}^{\prime}(e)=f_{i}^{\prime}(e)$, if $e \in E_{1}, i=1,2, \cdots, d$.It is easy to verify that $\left\{g_{1}^{\prime}, g_{2}^{\prime}, \cdots, g_{d}^{\prime}\right\}$ is a signed clique edge dominating family of $K_{\omega}$, so $d_{s c l}^{\prime}\left(K_{\omega}\right) \geq$ $d=d_{s c l}^{\prime}(G)$.

From two aspects above, we obtain that $d_{s c l}^{\prime}\left(K_{\omega}\right)=d_{s c l}^{\prime}(G)$.

Theorem 6. For any graph $G$ with size $m$, let $\omega(G)=\omega(\omega \geq 4)$ and the number of maximal cliques of $G$ be $\alpha(\alpha \geq 1)$, then 


$$
\begin{aligned}
& d_{s c l}^{\prime}(G)=\frac{\omega(\omega-1)}{2}, \text { if } \quad \omega \equiv 2 \operatorname{or} 3(\bmod 4) \\
& d_{s c l}^{\prime}(G)=\left\{\begin{array}{clll}
\frac{\omega(\omega-1)}{4}-1 & \text { if } \quad \omega \equiv 0(\bmod 4), \frac{\omega}{4} & \text { is } & \text { even } \\
\frac{\omega(\omega-1)}{4} & \text { if } \quad \omega \equiv 0(\bmod 4), \frac{\omega}{4} \quad \text { is } & \text { odd }
\end{array}\right. \\
& d_{s c l}^{\prime}(G)=\left\{\begin{array}{clll}
\frac{\omega(\omega+1)}{4}-1 & \text { if } \omega \equiv 1(\bmod 4), \frac{\omega}{4} \quad \text { is } & \text { even } \\
\frac{\omega(\omega+1)}{4} & \text { if } \omega \equiv 1(\bmod 4), \frac{\omega}{4} \quad \text { is } & \text { odd }
\end{array}\right.
\end{aligned}
$$

Proof. By the lemma4, we only consider its maximal clique of $G$. Let $K_{\omega} \in\left\{K_{\omega_{1}}, K_{\omega_{2}}, \cdots, K_{\omega_{\alpha}}\right\}$ be any maximal clique of $G$ and

$$
E\left(K_{\omega}\right)=\left\{e_{1}, e_{2}, \cdots, e_{\frac{\omega(\omega-1)}{2}}\right\}
$$

be an edge set of $K_{\omega}$. Therefore, a function $f^{\prime} \in F_{\text {sced }}(G)$ if only and if $f^{\prime}\left(E\left(K_{\omega}\right)\right) \geq 1$. We consider three cases as following.

Case 1: $\omega \equiv 2$ or $3(\bmod 4)$. When $\omega \equiv 2$ or $3(\bmod 4),\left|E\left(K_{\omega}\right)\right|=$ $\frac{\omega(\omega-1)}{2}$ is an odd number. Let $\frac{\omega(\omega-1)}{2}=2 p+1$ and $p$ be nonnegative integer.Define the signed clique edge dominating functions $f_{1}^{\prime}, f_{2}^{\prime}, \cdots, f_{\frac{\omega(\omega-1)}{2}}^{\prime}$ by $f_{i}^{\prime}\left(e_{i}\right)=f_{i}^{\prime}\left(e_{i+1}\right)=\cdots=f_{i}^{\prime}\left(e_{i+p}\right)=1$ and $f_{i}^{\prime}\left(e_{j}\right)=-1$ for every $e_{j} \in$ $E\left(K_{\omega}\right) \backslash\left\{e_{i}, e_{i+1}, \cdots, e_{i+p}\right\}, i=1,2, \cdots, \frac{\omega(\omega-1)}{2}, j=1,2, \cdots, \frac{\omega(\omega-1)}{2}$, where all subscripts are taken modulo $\frac{\omega(\omega-1)}{2}$. It is easy to see that $f_{i}^{\prime}\left(E\left(K_{\omega}\right)\right)=1$ for $i=1,2, \cdots, \frac{\omega(\omega-1)}{2}$ and $\sum_{i=1}^{\frac{\omega(\omega-1)}{2}} f_{i}^{\prime}(e)=1$ for each $e \in E\left(K_{\omega}\right)$. Hence

$$
\left\{f_{1}^{\prime}, f_{2}^{\prime}, \cdots, f_{\frac{\omega(\omega-1)}{2}}^{\prime}\right\} \in \Psi\left(K_{\omega}\right)
$$

and conclude that $d_{s c l}^{\prime}\left(K_{\omega}\right) \geq \frac{\omega(\omega-1)}{2}$. In view of theorem3, we obtain (2.1).

Case $2: \omega \equiv 0(\bmod 4)$. Let $\omega=4 k,(k$ is a positive integer $)$, then $\left|E\left(K_{\omega}\right)\right|=$ $\frac{\omega(\omega-1)}{2}=2 k(4 k-1)$ is an even number. We show that $d_{s c l}^{\prime}(G) \leq \frac{\omega(\omega-1)}{4}$ by theoreom3. We have two cases discuss the parity about $k(4 k-1)$.

Case $2.1 k(4 k-1)$ is odd. Define the family of signed clique edge dominating functions $f_{1}^{\prime}, f_{2}^{\prime}, \cdots, f_{k(4 k-1)}^{\prime}$ by $f_{1}^{\prime}\left(e_{1}\right)=f_{1}^{\prime}\left(e_{2}\right)=\cdots=f_{1}^{\prime}\left(e_{k(4 k-1)+1}\right)=1$ and $f_{1}^{\prime}\left(e_{j}\right)=-1$ for each $e_{j} \in E\left(K_{\omega}\right) \backslash\left\{e_{1}, e_{2}, \cdots, e_{k(4 k-1)+1}\right\}$, $f_{2}^{\prime}\left(e_{3}\right)=f_{2}^{\prime}\left(e_{4}\right)=\cdots=f_{2}^{\prime}\left(e_{k(4 k-1)+3}\right)=1$ and $f_{2}^{\prime}\left(e_{j}\right)=-1$ for each $e_{j} \in$ $E\left(K_{\omega}\right) \backslash\left\{e_{3}, e_{4}, \cdots, e_{k(4 k-1)+3}\right\}$,

$$
f_{\frac{k(4 k-1)-1}{2}}^{\prime}\left(e_{k(4 k-1)-2}\right)=f_{\frac{k(4 k-1)-1}{2}}^{\prime}\left(e_{k(4 k-1)-1}\right)=\cdots=f_{\frac{k(4 k-1)-1}{2}}^{\prime}\left(e_{2 k(4 k-1)-2}\right)
$$


$=1$ and $f_{\frac{k(4 k-1)-1}{2}}^{\prime}\left(e_{j}\right)=-1$ for each $e_{j} \in E\left(K_{\omega}\right) \backslash\left\{e_{k(4 k-1)-2}, e_{k(4 k-1)-1}\right.$, $\left.\cdots, e_{2 k(4 k-1)-2}\right\}$,

:

$f_{k(4 k-1)}^{\prime}\left(e_{2 k(4 k-1)-1}\right)=f_{k(4 k-1)}^{\prime}\left(e_{2 k(4 k-1)}\right)=\cdots=f_{k(4 k-1)}^{\prime}\left(e_{3 k(4 k-1)-1}\right)=1$ and $f_{k(4 k-1)}^{\prime}\left(e_{j}\right)=-1$ for each $e_{j} \in E\left(K_{\omega}\right) \backslash\left\{e_{2 k(4 k-1)-1}\right.$,

$\left.e_{2 k(4 k-1)}, \cdots, e_{3 k(4 k-1)-1}\right\}$, where all subscripts are taken modulo $\frac{\omega(\omega-1)}{2}$.

It is not hard to obtain $f_{i}^{\prime}\left(E\left(K_{\omega}\right)\right)=2$ for $i=1,2, \cdots, k(4 k-1)$ and $k(4 k-1)$

$\sum_{i=1} f_{i}^{\prime}(e)=1$ for each $e \in E\left(K_{\omega}\right)$,Hence, $\left\{f_{1}^{\prime}, f_{2}^{\prime}, \cdots, f_{k(4 k-1)}^{\prime}\right\} \in \Psi\left(K_{\omega}\right)$ and conclude that $d_{s c l}^{\prime}\left(K_{\omega}\right) \geq k(4 k-1)=\frac{\omega(\omega-1)}{4}$, so we obtain $(2.2)$.

Case 2.2: $k(4 k-1)$ is even. Define the family of signed clique edge dominating functions $f_{1}^{\prime}, f_{2}^{\prime}, \cdots$,

$f_{k(4 k-1)-1}^{\prime}$ by:

$f_{1}^{\prime}\left(e_{1}\right)=f_{1}^{\prime}\left(e_{2}\right)=\cdots=f_{1}^{\prime}\left(e_{k(4 k-1)+1}\right)=1$ and $f_{1}^{\prime}\left(e_{j}\right)=-1$ for each $e_{j} \in E\left(K_{\omega}\right) \backslash\left\{e_{1}, e_{2}, \cdots, e_{k(4 k-1)+1}\right\}$,

$f_{2}^{\prime}\left(e_{4}\right)=f_{2}^{\prime}\left(e_{5}\right)=\cdots=f_{2}^{\prime}\left(e_{k(4 k-1)+4}\right)=1$ and $f_{2}^{\prime}\left(e_{j}\right)=-1$ for each $e_{j} \in E\left(K_{\omega}\right) \backslash\left\{e_{4}, e_{5}, \cdots, e_{k(4 k-1)+4}\right\}$,

$f_{3}^{\prime}\left(e_{6}\right)=f_{3}^{\prime}\left(e_{7}\right)=\cdots=f_{3}^{\prime}\left(e_{k(4 k-1)+6}\right)=1$ and $f_{3}^{\prime}\left(e_{j}\right)=-1$ for each $e_{j} \in E\left(K_{\omega}\right) \backslash\left\{e_{6}, e_{7}, \cdots, e_{k(4 k-1)+6}\right\}$,

$f_{\frac{k(4 k-1)}{2}}^{\prime}\left(e_{k(4 k-1)}\right)=f_{\frac{k(4 k-1)}{2}}^{\prime}\left(e_{k(4 k-1)+1}\right)=\cdots=f_{\frac{k(4 k-1)}{2}}^{\prime}\left(e_{2 k(4 k-1)}\right)=1$ and $f_{\frac{k(4 k-1)}{2}}^{\prime}\left(e_{j}\right)=-1$ for each $e_{j}^{2} \in E\left(K_{\omega}\right) \backslash\left\{e_{k(4 k-1)}, e_{k(4 k-1)+1}, \cdots\right.$,

$\left.e_{2 k(4 k-1)}\right\}, f_{\frac{k(4 k-1)}{2}+1}^{\prime}\left(e_{k(4 k-1)+3}\right)=f_{\frac{k(4 k-1)}{2}+1}^{\prime}\left(e_{k(4 k-1)+4}\right)=\cdots$

$=f_{\frac{k(4 k-1)}{2}+1}^{\prime}\left(e_{2 k(4 k-1)+3}\right)=1$ and $f_{\frac{k(4 k-1)}{2}+1}^{\prime}\left(e_{j}\right)=-1$ for each $e_{j} \in E\left(K_{\omega}\right) \backslash$ $\left\{e_{k(4 k-1)+3}, e_{k(4 k-1)+4}, \cdots, e_{2 k(4 k-1)+3}\right\}$.

$\vdots$

$f_{k(4 k-1)-1}^{\prime}\left(e_{2 k(4 k-1)-1}\right)=f_{k(4 k-1)-1}^{\prime}\left(e_{2 k(4 k-1)}\right)=\cdots=$

$f_{k(4 k-1)-1}^{\prime}\left(e_{3 k(4 k-1)-1}\right)=1$ and $f_{k(4 k-1)-1}^{\prime}\left(e_{j}\right)=-1$ for each $e_{j} \in E\left(K_{\omega}\right) \backslash$ $\left\{e_{2 k(4 k-1)-1}, e_{2 k(4 k-1)}, \cdots, e_{3 k(4 k-1)-1}\right\}$, where all subscripts are taken modulo $\frac{\omega(\omega-1)}{2}$. Note that we have a jump of three in the arguments from $f_{1}^{\prime}$ to $f_{2}^{\prime}$ and from $f_{\frac{k(4 k-1)}{2}}^{\prime}$ to $f_{\frac{k(4 k-1)}{2}+1}^{\prime}$ and a jump of two in the subscripts.

It is a simple matter to obtain $f_{i}^{\prime}\left(E\left(K_{\omega}\right)\right)=2$ for $i=1,2, \cdots, k(4 k-1)-$ 
1.Furthermore, we have verified that $\sum_{i=1}^{k(4 k-1)-1} f_{i}^{\prime}\left(e_{k(4 k-1)+2}\right)=-1$ and

$$
\sum_{i=1}^{k(4 k-1)-1} f_{i}^{\prime}(e)=1
$$

for each

$$
e \in E\left(K_{\omega}\right) \backslash\left\{e_{k(4 k-1)+2}\right\}
$$

Hence

$$
\left\{f_{1}^{\prime}, f_{2}^{\prime}, \cdots, f_{k(4 k-1)-1}^{\prime}\right\} \in \Psi\left(K_{\omega}\right)
$$

and conclude that $d_{s c l}^{\prime}\left(K_{\omega}\right) \geq k(4 k-1)-1, \operatorname{sod}_{s c l}^{\prime}\left(K_{\omega}\right) \geq \frac{\omega(\omega-1)}{4}-1$, so we obtain (2.2) by the theoreom3.

Case 3: $\omega \equiv 1(\bmod 4)$. We can prove this case is true in same way of proof of Case 2.

\section{Acknowledgments}

This work is supported by the National Natural Science Foundation of P.R. China (61170302).

\section{References}

[1] B.Xu, On signed edge domination numbers of graphs, Discrete Math.239(2001),179-189. DOI: 10.1016/S0012-365X(01)00044-9

[2] B.Xu, Domination theory on graph,Science Press, China(2008). ISBN 9787-03-021910-7

[3] X.J.Li, J.M.Xu,The signed edge-domatic number of a graph,Graph and Combinatorics.29(2013),1881-1890. DOI 10.1007/s00373-012-1234-3

[4] S.Akbari, On the signed edge domination number of graphs, Discrete Mathematics.309(2009),587-594. DOI: 10.1016/j.disc.2008.08.021

[5] B.Xu, Two classes of edge domination in graphs,Discrete Applied Mathematics.154(2006),1541-1546. DOI: 10.1016/j.dam.2005.12.007 
[6] V.Lutz, Some remarks on the signed domatic number of graphs with small minimum degree,Applied Mathematics Letters.22(2009),1166-1169. DOI: 10.1016/j.aml.2008.09.006

[7] V.Lutz, B.Zelinka, Signed domatic number of a graph,Discrete Applied Mathematics.150(2005),261-267. DOI: 10.1016/j.dam.2004.08.010

[8] B.Xu, On signed cycle domination in graphs, Discrete Math.309(2009),1007-1012. DOI: 10.1016/j.disc.2008.01.007 